\title{
Powder flowability characterisation methodology for powder-bed- based metal additive manufacturing
}

\author{
A. B. Spierings ${ }^{1,3} \cdot$ M. Voegtlin ${ }^{1} \cdot$ T. Bauer ${ }^{1} \cdot$ K. Wegener $^{2}$
}

Received: 23 March 2015 / Accepted: 1 July 2015/Published online: 23 July 2015

(c) Springer International Publishing AG 2015

\begin{abstract}
Key in powder-bed-based additive manufacturing is the use of appropriate powder materials that fit to the process conditions. There are many parameters affecting the build process and the corresponding quality of the parts being built. Therefore, an accurate assessment of the powders becomes important. Such an assessment involves, besides others, the powder flowability, which should be sufficient in order to create good-quality powder layers. The current study aims at the development of suitable parameters and values for the qualification of metal powders for selective laser melting (SLM) with regard to their flowability. The powder flowability is assessed by the statistical analysis of the measured powder avalanche angles and the powder surface fractal, which give valuable information about the significance of inter-particle forces. A set of 21 different Fe- and Ni-based powders has been analysed and good correlations between the powder avalanche angles, the surface fractal and the particle shape with the optically evaluated flowability could be derived. The method allows a quantitative powder flowability assessment, which correlates with the experiences for powders for powder-bed-based additive manufacturing, especially for SLM.
\end{abstract}

A. B. Spierings

spierings@inspire.ethz.ch

1 Inspire-AG für mechatronische Produktionssysteme und Fertigungstechnik, Tannenstrasse 3, 8092 Zurich, Switzerland

2 Institute of Machine Tools and Manufacturing, ETH Zurich, 8092 Zurich, Switzerland

3 Inspire AG für mechatronische Produktionssysteme und Fertigungstechnik, Institute for Rapid Product Development Irpd, Lerchenfeldstrasse 5, 9014 St. Gallen, Switzerland
Keywords Additive manufacturing - Metal powders · Powder flowability · Powder properties

\section{Introduction}

Additive manufacturing (AM) is gaining more and more industrial attention, as the technologies involved such as selective laser sintering (SLS) for polymeric materials or selective laser melting (SLM), electron beam melting (EBM) and direct metal deposition (DMD) for metals allow the production of complex shaped parts. Such parts can significantly improve their performance in terms of, e.g. lightweight, functional integration, structurally optimisation or thermal behaviour. All these processes create the physical part to be built by slicing the CAD file of this part into several thin layers with a thickness typically between 20 and $200 \mu \mathrm{m}$, depending on the specific process and the processing conditions.

In SLS, SLM and EBM, thin layers of a powdered raw material are created, and an energy beam (laser, electron beam) is used to selectively scan the part's slices with the corresponding cross sections. This leads to full melting of the powder particles and to consolidation of the powder material, reaching almost $100 \%$ material density [1-4]. Much attention has been put in the development of processing windows for diverse plastic and metallic materials, and a wide range of materials and alloys are considered as "processable" [3-6]. The term "processability", however, contains several parameters, which affect the additive build processes and the final part properties (Fig. 1). This includes the physical and chemical properties of the raw material, the properties of the powders used and the powder layer creation device, typically a roller or a ruler (Fig. 4). These parameters have to be known and characterised in 
Fig. 1 Influences and their context to the SLM process

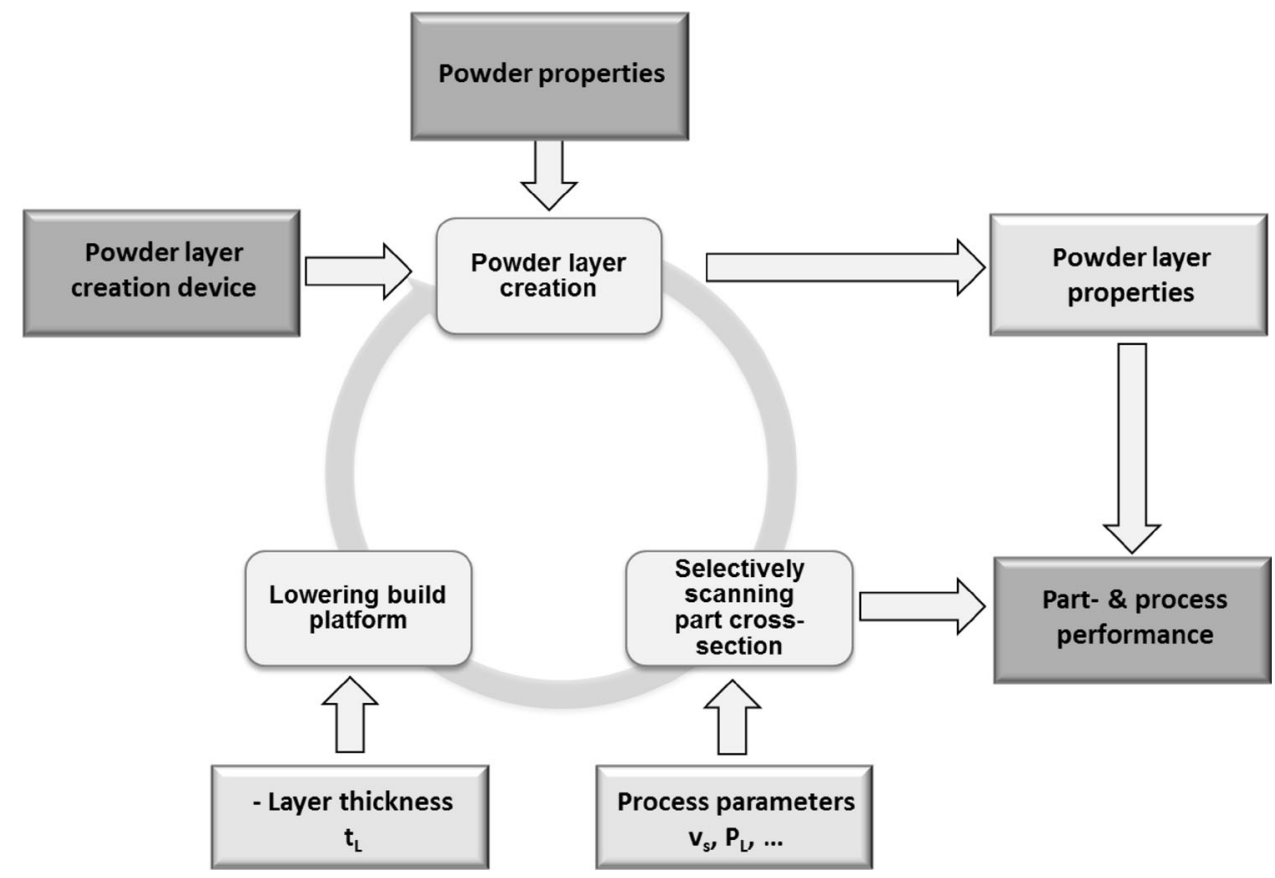

Fig. 2 Ishikawa diagram with influencing parameters for metal powders

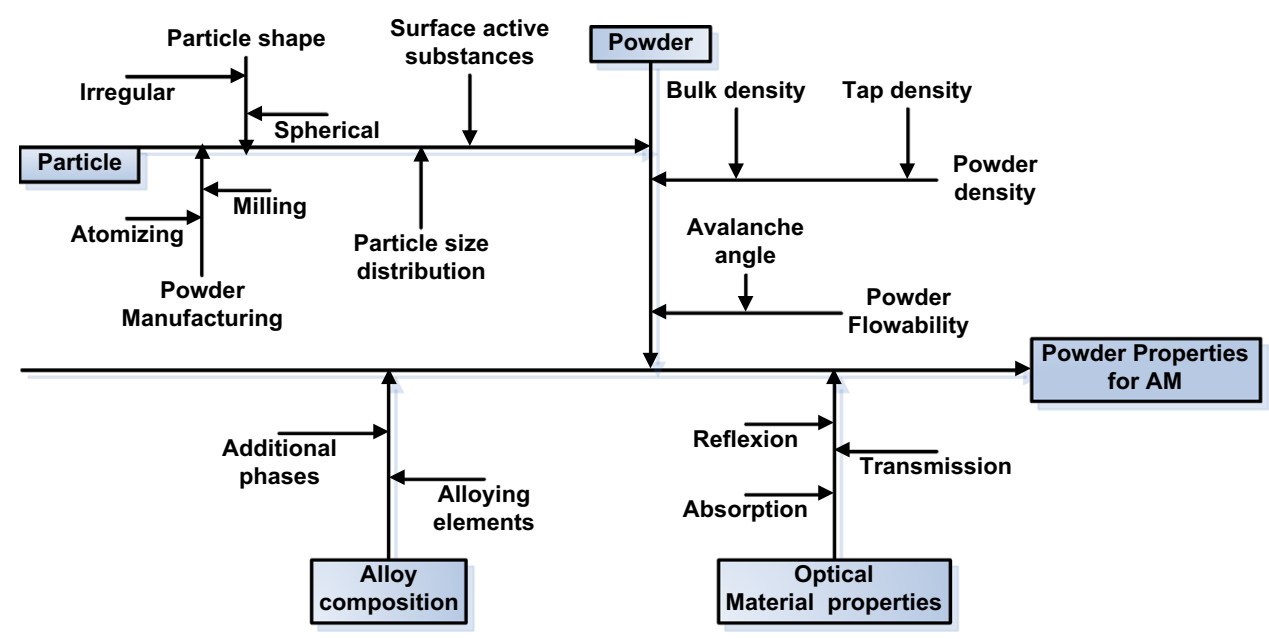

order to be able to optimise the build processes and the outcome, the parts.

Powder properties themselves include many different aspects such as the particle size distribution and related powder density (bulk and tap density) as well as flowability, which directly affect the layer generation capabilities. Furthermore, the optical and thermal properties are also affected by these parameters (Fig. 2).

Having generated a thin powder layer using a certain coating device, the properties of this powder layer then will be different to bulk powder, as, e.g. wall effects affect the properties of the final powder layer (Fig. 3).

The layer properties directly affect the processing window, e.g. by changed laser absorption properties, as indicated by Boley [7]. Additionally, Gürtler [8] has shown the influences of particle size distributions (PSD) and powder bed quality on melt pool dynamics and consequently on porosity. Today, and especially for AM where very thin powder layers are required with "good" layer properties, there is a tendency to use as fine powders as possible to improve the processing window (scan speed) $[8$, 9] and consequently the microstructure, part density and surface qualities. However, this increases the risk to process powders with an insufficient flowability and correspondingly ends up with a bad layer quality. Inter-particle forces $F_{\mathrm{i}}$ such as Van der Waals attractive forces, as well as gravitational forces $F_{\mathrm{g}}$ and eventually moisture can influence the behaviour of a powder [10]. Krantz [11] reports 
Fig. 3 Powder layer properties, dependent on powder density and flowability

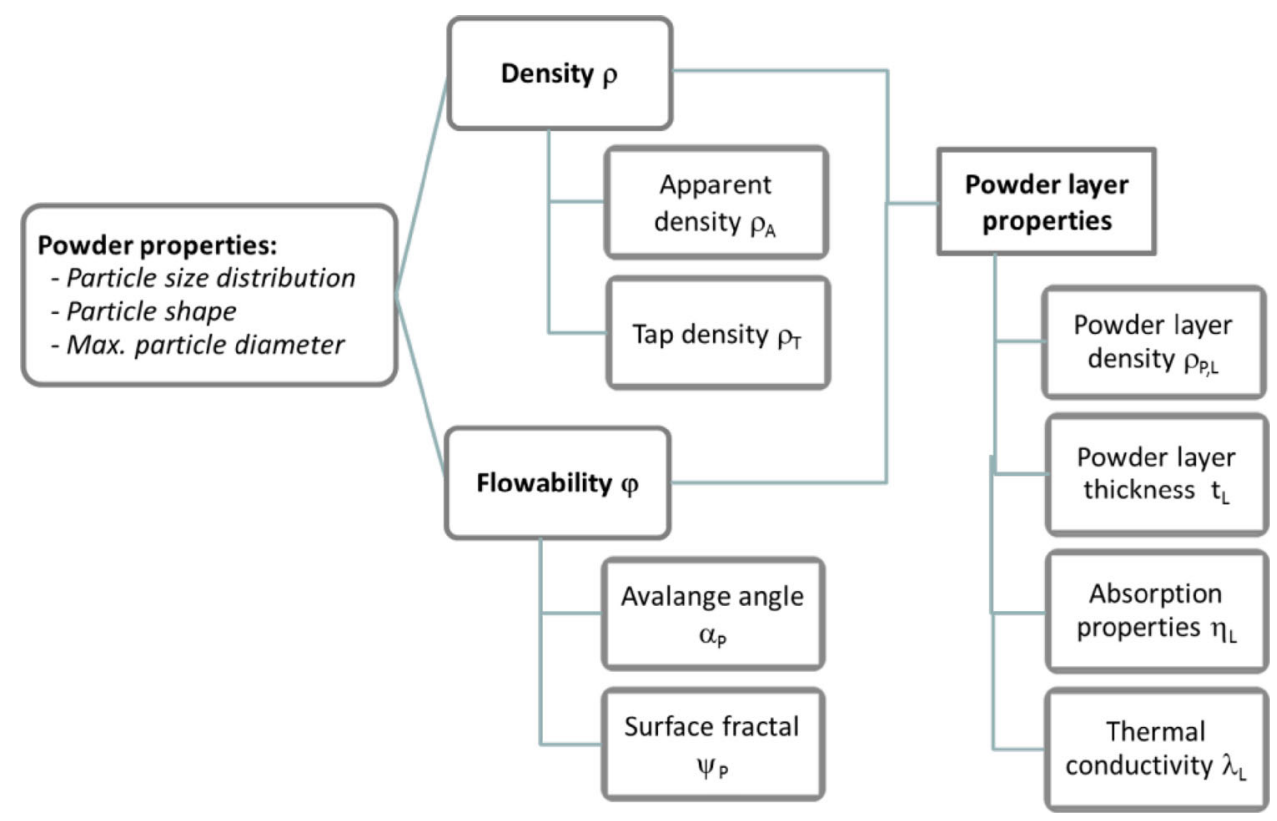

that Van der Waals forces can account for up to 80-90\% of inter-particle forces and Castellanos [12] defined the cohesive granular bond number $\mathrm{BN}=F_{\mathrm{i}} / F_{\mathrm{g}}$ to divide powders into cohesive and non-cohesive powders. Powders according to [13] tend to agglomerate the finer they are, as $\mathrm{BN}$ becomes high and consequently resulting in poor powder flowability. Yu et al. [13] concluded in their analyses of the packing densities of alumina and silicon carbide powders that the Hausner ratio HR seems to be a useful parameter reflecting particle-particle friction. The question remains open how a particle size distribution should look like for an optimal powder layer quality and how many fine particles are allowed to still have sufficient powder flowability.

Generally, it is essential to characterise powders in a manner that is as close to the manufacturing process as possible. In that respect, a good flowability of a powder is required for a successful AM process. This is, however, not sufficient, as from the characterisation of powder flowability no information is derived whether, e.g. the particle sizes and their distribution are optimal for AM, e.g. regarding powder layer density or its optical properties (laser absorption). For that purpose, further requirements have to be derived.

The current paper focuses on an adequate powder flowability characterisation technique for powder-bedbased metal AM (SLM) processes. The derived flowability value is correlated with an experience-based optical flowability evaluation, which today is most often applied to assess the basic "processability" of a powder for SLM.

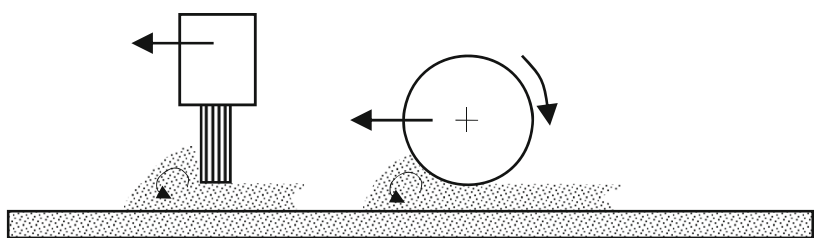

\subsection{Review on powder flowability measurement techniques}

A comprehensive overview on different techniques for powder flow measurement is given by Schulze [14, 15] and Krantz [11]. Krantz concluded that it is essential to apply a measurement technique that is as close to the final process as possible, especially with a view on the stress state in the powder. Therefore, some techniques are presented and discussed before the needs for AM processes, where comparably small amounts of powders are used to create thin powder layers, and where powders are more or less dynamically moved over the build area. More precisely, the evaluation is focusing on AM processes where the powder layers are created by a ruler or a rotating cylinder (Fig. 4) and where the powder is taken from a powder reservoir. In such a situation, the powders have a comparably high free surface and can be aerated to some degree depending on the speed of the coating device.

\subsubsection{Ring shear cell tester}

For bulk powders, the ring shear cell tester (ASTM D6773 [16], Fig. 5) is often used and industrial standard to get
Fig. 4 Schematic of AM coating processes 


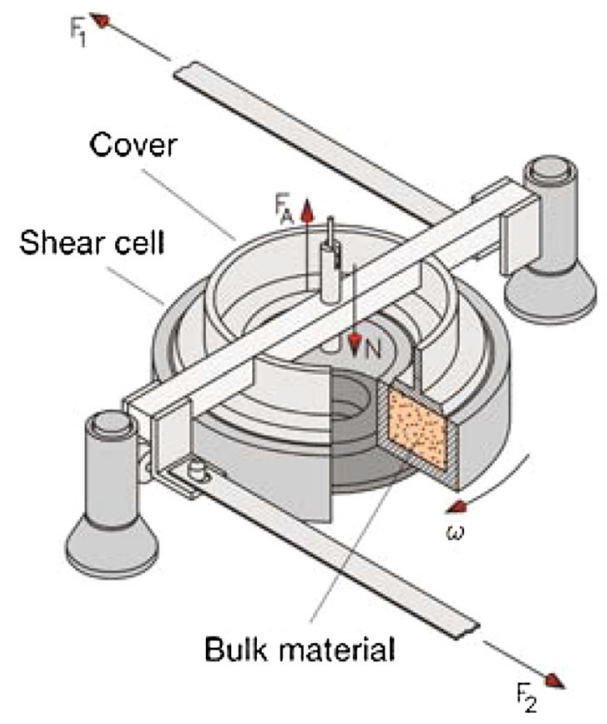

Fig. 5 Ring shear tester, type Schulze RST-01 [17]

insight into powder properties such as powder flowability, compressive strength, powder compressibility, consolidation time ("cacking"), internal and wall friction, and bulk density. A very good description of the methodology has been given by Schulze [17]. For the use for powders in AM technologies and especially where powders are moved over a powder bed, this technique seems to be not well suited as the powders are assessed under a compressive load, which is far away from the situation in AM.

\subsubsection{Hausner ratio $H R$}

The Hausner ratio HR, defined as the ratio between tapped and bulk density [18], is a common technique and widely used. The measurement of powder density (apparent/bulk and tap density) is described in ASTM D7481-09 [19]. Yu et al. [13] concluded that the HR can be used to describe the packing behaviour of powders when they are subjected to tapping. Abdullah reports that powders with HR $\leq 1.25$ are considered as freely flowing and that cohesive and nonflowing powders show a HR $>1.40$. In the field of AM, Zocca [20] used HR as the flowability indicator for bulk ceramic powders. However, there are some drawbacks associated with HR, especially when compared to the situation in AM where thin powder layers are created and no compression or tapping is applied. Therefore, own experiences show that HR does not nicely correlate with an optical evaluation of flowability and that different powders are not well differentiated, which is supported by Soh [21], who further reports that HR does not nicely correlate with more sophisticated measurement techniques. Furthermore, although the measurement of bulk and tap density is comparably easy, especially for the tap density it is well known that powders often do not reach stable density values within a reasonable number of tapping cycles [22]. Miyajima [23] showed that the change in void volume fraction of packed powder beds can be estimated, but it might be necessary to apply $>100$ taps to reach a more or less stable situation depending on particle shape. The dependence of HR on particle shape was also approved by Zou and $\mathrm{Yu}$ [24]. They showed that for large non-cohesive non-spherical powders HR decreased with an increase in sphericity. The fact that tapped density is highly dependent on the number of tapping was also approved by Abdullah [25]. Rastogi et al. [22] analysed the HR of ultrafine coals and found that even after $>1000$ taps the ratio did not reach a steady state. Furthermore, measuring bulk and tap powder densities is comparably far away from the situation in a powder-bed-based AM process (SLM), where small amounts of powders are more or less "dynamically" put on the built platform and on the last already solidified layer of the part. Therefore, as it is necessary to apply a flowability characterisation method allowing the comparison of as different powders as possible, HR is not considered to be ideal for application in AM.

\subsubsection{Angle of repose/Hall flowmeter}

The angle of repose defined in ISO-4490 [26] /ASTM B213 [27] is an easy principle, where powder flows freely through a funnel onto a plate and the slope angle of the developed cone to the base plate is the angle of repose and considered as a measure for powder flowability. The method is also recommended by ASTM as the characterisation method for metal powders for AM [28]. Alternatively, the time required to discharge the powder can be used as a measure for flowability, as commented by Schulze [15]. For freely flowing powders, a low angle (and short discharge time) can be expected as avalanches can easily flow down the slope, whereas for cohesive powders this angle will be high. However, Schulze [15] also concluded that the methodology yields to a quantitative statement about the powder's flowability but that the operators filling method can influence the results. In addition, the principle does have the drawback that for some (for the use in AM still sufficiently flowing) powders the $0.1^{\prime \prime}$-diameter of the opening is too small in order for them to flow through the funnel. This makes it difficult to quantitatively assess different powders, although the methodology can be considered as closer to the AM processing conditions than other techniques. However, as the stress state of the powder in the developed powder cone is still different to the situation in SLM or SLS (Fig. 4), this technique is not considered as best suited. 


\subsubsection{Avalanche angle}

The measurement of the avalanche angle is as a methodology, which is nearer to powder-bed-based AM (SLS, SLM) than the other techniques. It typically consists of a rotating, transparent drum filled with a certain amount of powder and a camera in front of a backlight. The camera records pictures of the powder free surface and the cross-sectional area of powder inside the drum. The pictures can be analysed for different values associated with powder flowability. Soh [21] suggested to analyse pharmaceutical powders based on their avalanching behaviour and proposed to quantify powders based on the avalanche flow index (AFI) and cohesive interaction index (CoI). The methodology has also been used by Krantz [11] to assess polyurethane and polyesterepoxy powders and he found a good correlation between the angle of repose and the measurement of the avalanche angle, as both methods subject the powders to similar stress states. Gu [29] used the avalanche angle (he called this measurement "angle of repose") to characterise three different Ti6Al4V powders with regard to influences on microstructure and tensile strength of SLM built parts. However, he rotated the drum manually three times, which does not give any indication about the statistical variations of the angle. Amado et al. [30] applied the methodology successfully to a range of plastic powders for SLS. He concluded that other more conventional test methods are less suitable to assess powders for AM. The avalanche test allows a statistical analysis and extraction of distribution characteristics of quite a number of different parameters like avalanche angle, surface fractal, volume expansion rate and many more. Based on the positive experiences with the avalanche test, we analyse a range of different atomised metal powders, basically suitable to be used in SLM, for their flowability.

\section{Materials and methods}

\subsection{Powders}

Most of the powders used in SLM today are gas or water atomised and therefore more or less spherical in shape. A range of different atomised nickel and iron powders have been selected (Table 1). Most of the mono-modal distributions follow a log-normal distribution, except for Fe4, which follows more a normal distribution. The PSD of the selected powders were measured using the PowderShape ${ }^{\mathrm{TM}}$ particle analysis system described below.

Powders Fe7, Fe12-Fe14 and Ni7 are bi-modal, with a significant amount of fine particles besides a main peak for higher particle sizes.

\subsection{Powder particle size distribution}

The PowderShape ${ }^{\mathrm{TM}}$ system analyses optically several thousand single particles (typically $>10,000$ ) with an appropriate filtering mask making sure that single particles are analysed and no agglomerates of particles. According to Dvorak [31], the method delivers comparable results as laser scattering methods. For this study, the selected particles are having a diameter between 4 and $75 \mu \mathrm{m}$, thereby covering the whole particle size range of the powders and the single particles are considered as coextensive circle diameters. The PSD's are mono- or bi-modal, whereas the most of the mono-modal powders can be described by a log-normal distribution. The optical analysis of particles enables not only to calculate particle size, but also parameters related to the particle shape. Besides the particle size, also the ellipticity $E$ of the particles has been analysed, as this can give additional information about powder flowability.

\subsection{Powder flowability measurement}

Powder flowability is of course not the only parameter affecting the processability of a powder. However, a powder does at least need to flow sufficiently in order to generally enable its additive processing in SLM or SLS. The requirements for flowability are thereby dependent on the machine concept, and mostly influenced by the type of coating device, whether it is a ruler or a rotating drum, respectively, and if powder is taken from a powder reservoir next to the build plate or if the powder needs to flow through a funnel into a coating device. Therefore, the flowability behaviour is considered as an important parameter affecting also the (SLM) process, and the results of the final product and an appropriate assessment become essential. To analyse the powder flowability $F$, two methods have been applied. The first one is an optical evaluation, which is the typical way how powders are qualified today for SLM with regard to flowability $\left(\varphi_{\text {opt }}\right)$. The second one is a quantitative evaluation of flowability $\left(\varphi_{\text {cal }}\right)$ based on the avalanche angle and the surface fractal of the powders, measured using Revolution Powder Analyzer (Mercury Scientific Inc., Newtown CT).

\subsubsection{Optical evaluation $\varphi_{\text {opt }}$}

An optical evaluation of the flowability $F$ of the powders under investigation has been performed based on an independent assessment of five experienced people. The evaluation follows a classification between 1 (very good flowability) and 5 (insufficient flowability for SLM, high tendency for agglomeration) as shown in Table 2. A value between 2.5 and 3.0 is considered as the limit range for 
Table 1 List of iron- (left) and nickel-based (right) powders and $D_{10}, D_{50}$ and $D_{90}$ quantiles (volume based)

\begin{tabular}{lcllll}
\hline Powder no. & $D_{10}(\mu \mathrm{m})$ & $D_{50}(\mu \mathrm{m})$ & $D_{90}(\mu \mathrm{m})$ & Ellipticity $(-)$ & PSD-distribution \\
\hline Fe1 & 12.7 & 23.9 & 41.4 & $1.36 \pm 0.27$ & Log-normal \\
Fe2 & 9.8 & 27.0 & 50.2 & $1.44 \pm 0.29$ & Log-normal \\
Fe3 & 9.6 & 24.5 & 46.2 & $1.46 \pm 0.31$ & Log-normal \\
Fe4 & 23.1 & 30.2 & 41.0 & $1.22 \pm 0.22$ & Normal \\
Fe5 & 7.2 & 16.9 & 29.0 & $1.57 \pm 0.38$ & Log-normal \\
Fe6 & 10.8 & 21.5 & 39.0 & $1.38 \pm 0.27$ & Log-normal \\
Fe7 & 5.2 & 8.5 & 29.4 & $1.38 \pm 0.43$ & Bi-modal \\
Fe9 & 9.9 & 18.1 & 35.1 & $1.34 \pm 0.26$ & Log-normal \\
Fe10 & 14.6 & 34.7 & 59.5 & $1.39 \pm 0.27$ & Log-normal \\
Fe12 & 22.0 & 37.3 & 53.9 & $1.25 \pm 0.24$ & Bi-modal \\
Fe13 & 26.9 & 43.0 & 55.8 & $1.37 \pm 0.30$ & Bi-modal \\
Fe14 & 25.2 & 37.0 & 54.9 & $1.41 \pm 0.51$ & Bi-modal \\
Ni1 & 7.8 & 18.1 & 34.6 & $1.51 \pm 0.45$ & Log-normal \\
Ni2 & 9.0 & 19.4 & 33.9 & $1.55 \pm 0.41$ & Log-normal \\
Ni3 & 13.4 & 27.6 & 51.9 & $1.36 \pm 0.31$ & Log-normal \\
Ni4 & 15.2 & 33.2 & 55.6 & $1.30 \pm 0.26$ & Log-normal \\
Ni5 & 12.2 & 25.2 & 40.3 & $1.28 \pm 0.26$ & Log-normal \\
Ni6 & 11.3 & 25.2 & 44.4 & $1.56 \pm 0.44$ & Log-normal \\
Ni7 & 28.3 & 35.4 & 42.8 & $1.35 \pm 0.41$ & Bi-modal \\
Ni8 & 12.3 & 27.5 & 46.2 & $1.42 \pm 0.29$ & Log-normal \\
Ni9 & 11.1 & 21.8 & 37.3 & $1.35 \pm 0.30$ & Log-normal \\
\hline & & & &
\end{tabular}

Table 2 Optical classification of powder flowability $\varphi_{\text {opt }}$

\begin{tabular}{|l|l|l|l}
\hline $\begin{array}{l}\varphi=1 \text { - very good flowability } \\
\text { no agglomerations }\end{array}$ & $\begin{array}{l}\varphi=2 \text { - sufficient flowability } \\
\text { Very loose agglomerations }\end{array}$ & $\begin{array}{l}\varphi=3 \text { - critical flowability } \\
\text { loose agglomerations }\end{array}$ & $\begin{array}{l}\varphi=4-\text { unsufficient flowability } \\
\text { severe agglomerations }\end{array}$ \\
\hline & & & \\
& & &
\end{tabular}

their usability in additive processing machines equipped with a ruler and an additional powder reservoir next to the build plate (e.g. Concept Laser machines); a reasonable limit is 2.5 , ensuring that the powder flows sufficiently to realise good-quality powder layers. However, for other machine configurations, this limit value might be different.

\subsubsection{Revolution Powder Analyzer}

The Revolution Powder Analyzer works according to Fig. 6. It consists of a rotating drum with an inner diameter of $100 \mathrm{~mm}$ and a width of $35 \mathrm{~mm}$, covered on both sides with transparent glass. A camera records pictures of the rotating drum (0-200 $\left.\mathrm{min}^{-1}\right)$ before a backlight. As the powder occupies a certain area, the record is a black-andwhite picture of the powder and the avalanches, respectively, within the drum (Fig. 6, left). Two different tests are applied: Fluidisation test and flowability test. The fluidisation cycle is used to put the powder into a normalised condition where effects of consolidation and handling are removed (see Table 3).

\subsubsection{Measurement parameters}

The avalanche angle $\left(\alpha_{\mathrm{P}}\right)$ is the angle of a linear regression of the free powder surface just before an avalanche starts, 
Fig. 6 Schematic of the Revolution Powder Analyzer principle
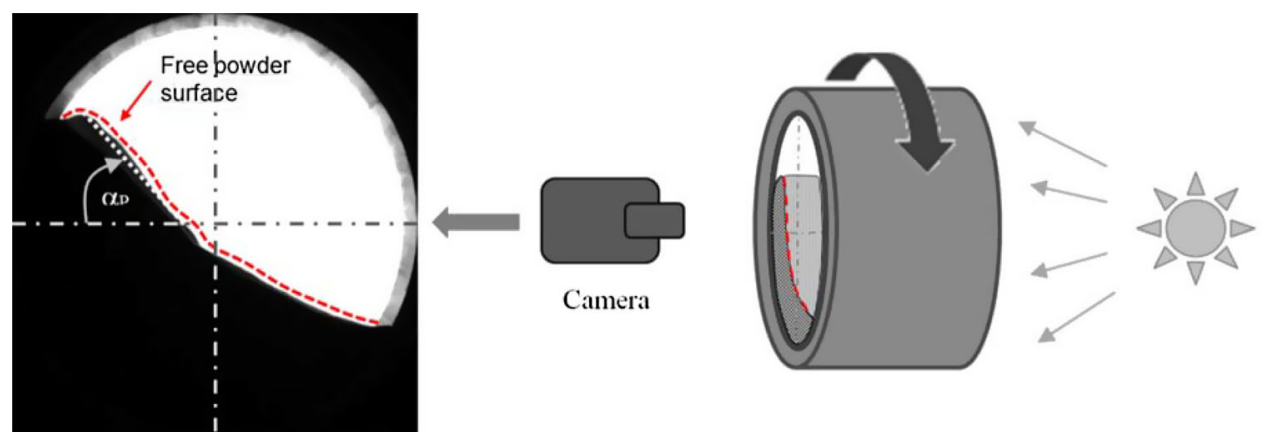

Table 3 Main parameters for fluidisation and flowability test for metal powders

\begin{tabular}{ll}
\hline Step 1 powder preparation by fluidisation & \\
Powder sampling volume & $99.4 \mathrm{~cm}^{3}$ (tap density) \\
Prep rate/time & $90 \mathrm{rpm} / 30 \mathrm{~s}$ \\
Start rate & $40 \mathrm{rpm}$ \\
End rate & $90 \mathrm{rpm}$ \\
Step rate & $10 \mathrm{rpm}$ \\
Step 2 measurement of flowability of the powder \\
Powder sampling volume & $99.4 \mathrm{~cm}^{3}$ (tap density) \\
Prep time & $30 \mathrm{~s}$ \\
Rotation rate & $0.6 \mathrm{rpm}$ \\
Imaging rate & $15 \mathrm{fps}$ \\
Angle calculation & $1 / 2 \emptyset_{\text {Drum }}$ \\
Avalanche threshold & $0.65 \%$ \\
No. of recorded avalanches & $3 \times 128$ \\
\hline
\end{tabular}

measured to a horizontal line. In order to improve the quality of the measurements, the software only analyses the left half side of the drum.

The avalanche surface fractal $\left(\psi_{\mathrm{P}}\right)$ is a measure of the fractal dimension of the surface profile. The theory of fractal structures is going back to Richardson and well described by Allen et al. [32]. It is basically the description of the real length $L$ between two points on the powder surface by the following Eq. (1):

$L(\varepsilon)=M \times \varepsilon^{(1-D)}$

with $\varepsilon$ the scale of the measurement, $M$ and $D \geq 1$ are constants. For the calculation of $M$ and $D$, the scale $\varepsilon$ is varied from $\varepsilon_{\min }$, which is defined by the resolution of the recorded image (pixel size), and $\varepsilon_{\max }=1 / 3 \varnothing_{\text {Drum }}$. As for different scales $\varepsilon$, the real length $L(\varepsilon)$ is different, $M$ and $D$ can be fitted over $\varepsilon$. For a perfectly smooth surface (with $D=1), M=L$ and therefore the shortest distance between two points. According to Richardson [32], " $D$ is a 'characteristic' of a frontier, (which) may be expected to have some positive correlation to with one's immediate visual perception of the frontier". As $D$ is typically a very small value, the $\psi_{\mathrm{P}^{-}}$-value recorded by the software is 100 times $D$ to improve readability. This fractal concept does basically fit to the perception of a powder whether it is cohesive, forming agglomerates, or not, and is therefore an indirect measure of inter-particle forces.

The volume expansion ratio $(\phi)$ has been well described by Amado [30] as the ratio between the volume measured inside the drum and the volume occupied by the powder in the sample preparation container. For metal powders, a preparation container with a volume of $99.373 \mathrm{~cm}^{3}$ is used. This container is filled with powder and manually tapped until no more powder can be filled in. This amount of powder is then put into the drum. By rotating the drum, the powder will aerate to some degree and will therefore tend to the apparent or bulk powder density. The comparison of this powder volume (measured as the surface of the projection of the occupied volume multiplied with the width of the drum) with the volume of the container $\phi$ can be related to the HR with the improvement that the measurement is less sensitive to manual operations.

\subsection{Log-Normal distribution}

The particle diameters $(x)$, the avalanche angle $\left(\alpha_{\mathrm{P}}\right)$ and the surface fractal $\left(\psi_{\mathrm{P}}\right)$ follow in most cases a log-normal distribution density function $f(x)$

$f(x)=\frac{1}{\sqrt{2 \pi} \times \sigma \times x} \times e^{-\frac{(\ln (x)-\mu)^{2}}{2 \times \sigma^{2}}}$

with the characteristic moments $\mu$ and $\sigma$, corresponding to the mean value $\bar{x}$ and the standard deviation $\mathrm{s}$ in the Normal distribution. The $\alpha_{\mathrm{P}^{-}}$and $\psi_{\mathrm{P}^{-}}$-distributions have been fitted for the log-normal distribution with the $\mathrm{JMP}^{\circledR}$ V10.0.0 software package. Out of this distribution, it is possible to calculate the arithmetic mean $E(f(x))$ and the variance $\operatorname{Var}(f(x))$ by 


$$
\begin{aligned}
E(f(x))= & \frac{1}{\sqrt{2 \pi} \sigma} \times \int_{0}^{+\infty} x \times \frac{e^{-\frac{(\ln (x)-\mu)^{2}}{2 \times \sigma^{2}}}}{x} \mathrm{~d} x=e^{\mu+\frac{\sigma^{2}}{2}} \\
\operatorname{Var}(f(x)) & =\frac{1}{\sqrt{2 \pi} \sigma} \times \int_{0}^{+\infty}\left(x-e^{\mu+\frac{\sigma^{2}}{2}}\right) \times \frac{e^{-\frac{(\ln (x)-\mu)^{2}}{2 \times \sigma^{2}}}}{x} \mathrm{~d} x \\
& =e^{2 \mu+\sigma^{2}} \times\left(e^{\sigma^{2}}-1\right) \\
& =E^{2}(f(x)) \times \operatorname{Var}^{2}(f(x))
\end{aligned}
$$

with $\operatorname{Var} K(f(x))=\sqrt{\left(e^{\sigma^{2}}-1\right)}$

\section{Results and discussion}

The evaluations in this chapter are following the aim to derive a quantitative assessment of the flowability $\varphi_{\text {cal }}$, allowing offline evaluation of powders for AM. This is done by comparing the optically evaluated powder flowability $\varphi_{\text {opt }}$ with the Hausner ratio HR, the volume expansion ratio $\phi$, and powder Avalanche angle $\left(\alpha_{\mathrm{P}}\right)$ and the surface fractal $\left(\psi_{\mathrm{P}}\right)$, respectively.

\subsection{Optical evaluation of flowability $\varphi_{\mathrm{opt}}$}

The optical evaluation of powder flowability $\varphi_{\text {opt }}$ shows that the powders investigated flow very differently (Fig. 7). The mean of the standard deviation of $\varphi_{\text {opt }}$ of all powders is 0.36. The comparison to the often used Hauser ratio HR, which is shown in Fig. 7 indicates that for fine metal powders HR does not sufficiently indicate powder flowability - most of the powders would be considered as freely flowing ( $\mathrm{HR}<1.25)$, which is obviously not the case ( $\varphi_{\text {opt }}>2.5$ for several powders). The volume expansion ratio $\phi$, however, does explain flowability better, but it seems not to be fully sufficient to quantify flowability as there is still significant scatter in Fig. 7 (right). The variations shown in Fig. 7 (right) indicate that other effects may play a significant role, such as inter-particle forces etc. It becomes therefore clear that more sophisticated assessment methods are required.

As from an industrial point of view, one is not able to analyse inter-particle forces etc., methods such as the measurement of $\alpha_{\mathrm{P}}$ and $\psi_{\mathrm{P}}$ seem to be more suited as these parameters are a phenomenological measure of such effects.

\subsection{Avalanche angle $\alpha_{P}$ and surface fractal $\psi_{P}$}

Figure 8 presents the cumulative size distribution of the avalanche angle for all powders. A similar picture is obtained when plotting the avalanche surface fractal $\psi_{\mathrm{P}}$ instead of the avalanche angle $\alpha_{P}$. It shows the partially wide scatter of the flowability parameters. Some of the avalanche angles reach values of up to $70^{\circ}$ (e.g. Fe11), whereas for other powders all avalanches angle are $<45^{\circ}$ (e.g. Fe14).

The direct comparison of the optical evaluation with the measured $\alpha_{\mathrm{P}}$ and $\psi_{\mathrm{P}}$ values (Fig. 9) shows that there is a clear relationship between the $\varphi_{\text {opt }}$ and $\alpha_{\mathrm{P}}$ and $\psi_{\mathrm{P}}$. In addition, it also seems to be dependent on the base of the alloys investigated (Fe, Ni). Both observations support the expectation that inter-particle forces play a significant role. The variation in $\alpha_{\mathrm{P}}$ and $\psi_{\mathrm{P}}$, however, is significant, which indicates that besides inter-particle forces also the specific particle size distribution and eventually the shape of the particles will play an important role. It is well known that a high amount of fine particles does negatively affect the flowability of a powder, as for fine particles the cohesive granular bond number (BN) [12] becomes high.

So far, only the mean values for $\alpha_{\mathrm{P}}$ and $\psi_{\mathrm{P}}$ have been used to correlate powder flowability. A "good" powder,
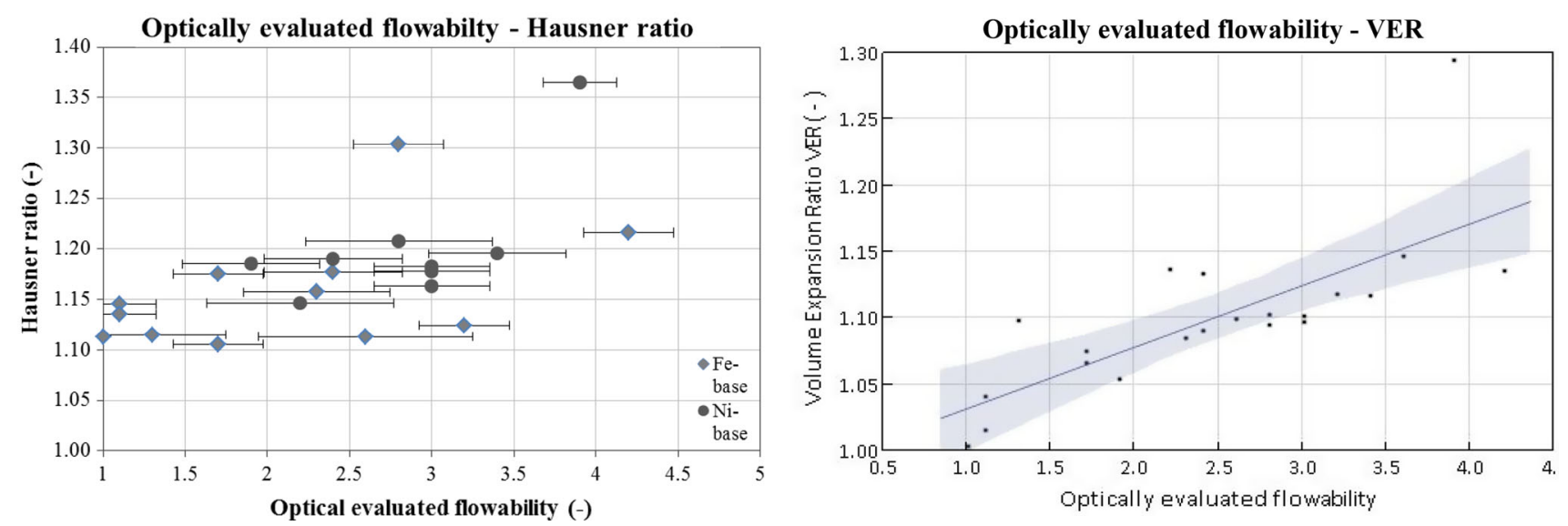

Fig. 7 Comparison of optical flowability rating with HR and $\phi$ 

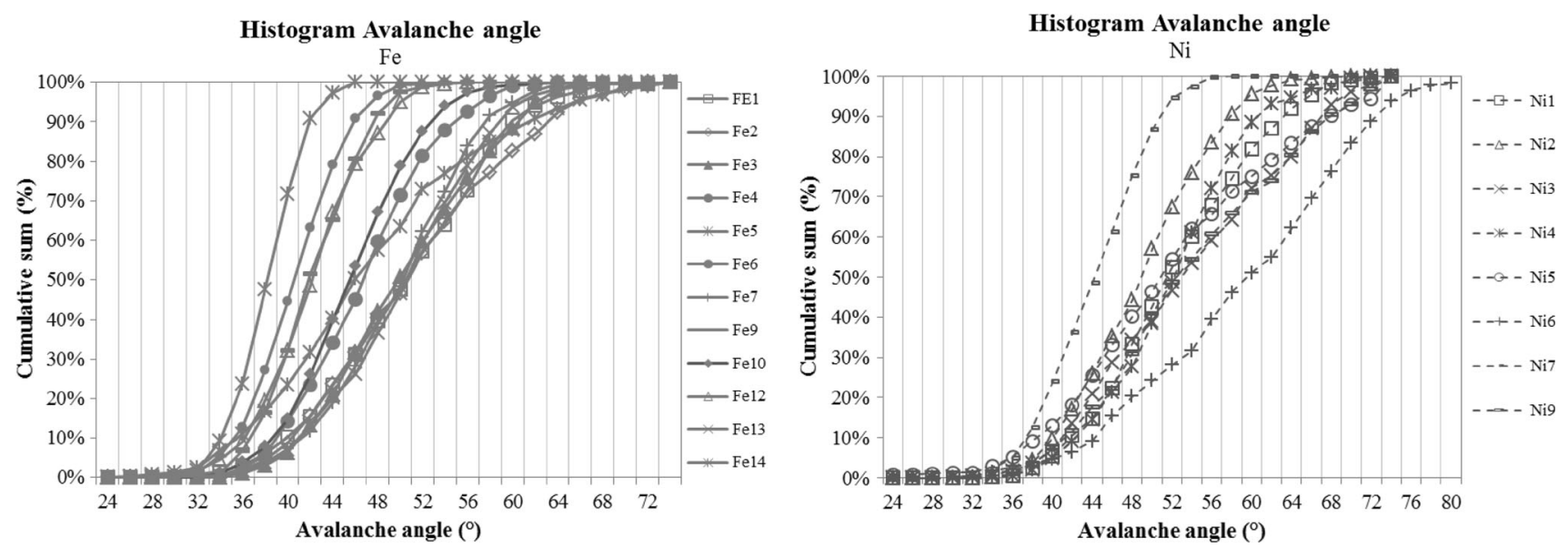

Fig. 8 Cumulative size distribution of the avalanche angle $\alpha_{\mathrm{P}}$ for Fe (left) and Ni alloys (right)

Fig. 9 Dependence of $\varphi_{\text {opt }}$ on $\alpha_{\mathrm{P}}$ and $\psi_{\mathrm{P}}$ for both alloy bases

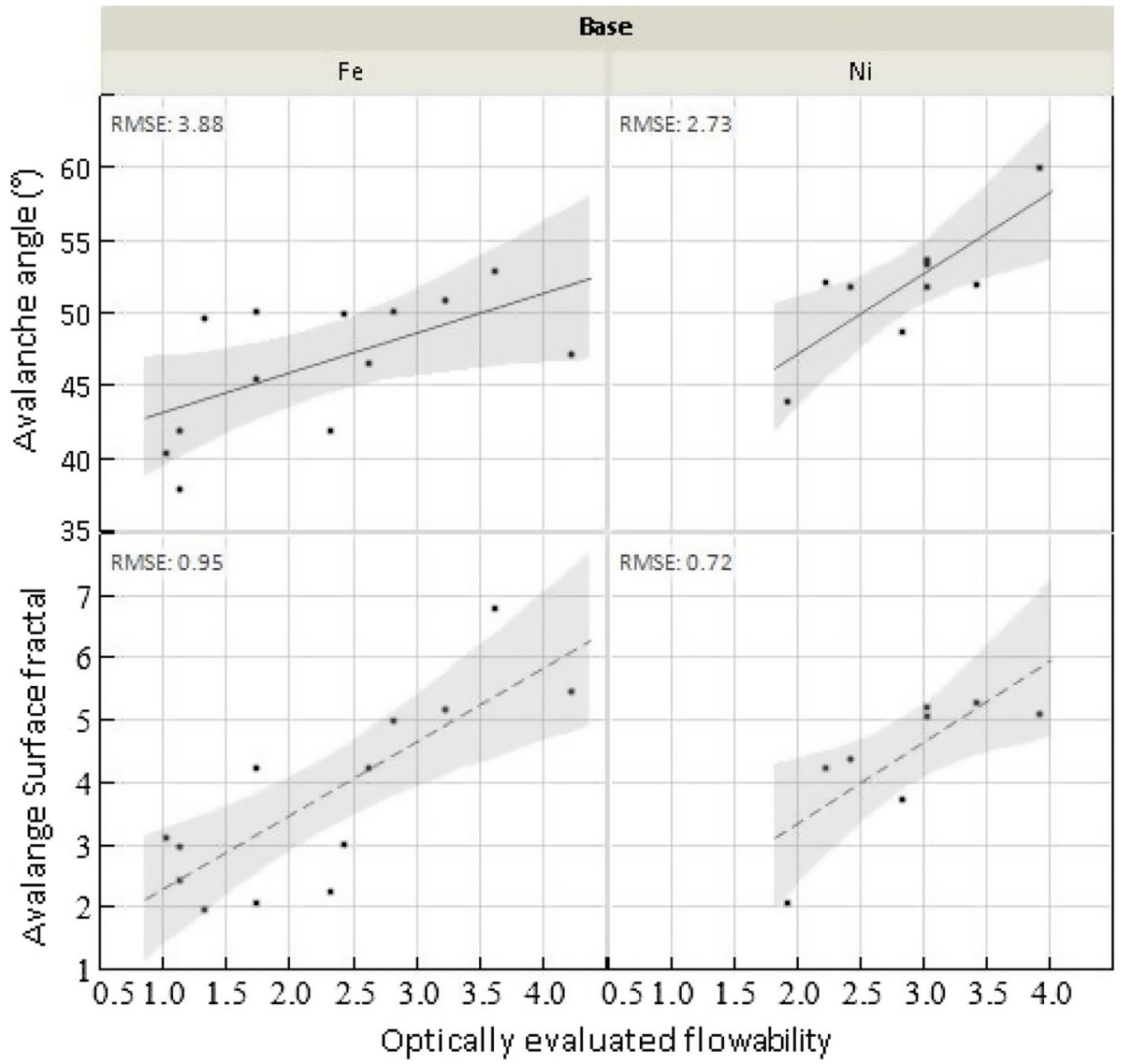

however, will not only have low values of $\alpha_{\mathrm{P}}$ and $\psi_{\mathrm{P}}$, but will also have a narrow distribution of $\alpha_{\mathrm{P}}$ and $\psi_{\mathrm{P}}$. This is because one can expect that the presence of agglomerations would lead to a more irregular occurrence of avalanches and a more jagged powder surface (higher $\psi_{\mathrm{P}}$-values). It is therefore worth considering the distribution of $\alpha_{\mathrm{P}}$ and $\psi_{\mathrm{P}}$ to better explain powder flowability. Figure 10 presents the avalanche angles of the powders compared to their coefficient of variation $\operatorname{Var} K$ from Eq. (4), showing a clear (second-order polynomial) relationship, where low avalanche angles are associated with a low $\operatorname{Var} K$. It indicates further that additional information can be achieved by considering the "width of the distribution".

In order to better be able to derive requirements and limits for powders, the variance of the avalanche angle $\operatorname{Var}\left(\alpha_{\mathrm{P}}\right)$ from (4) is compared to the avalanche surface 


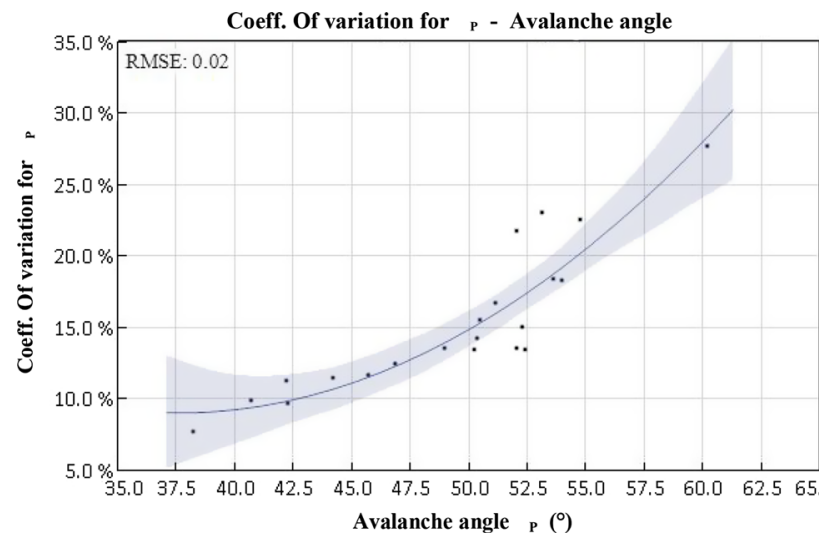

Fig. 10 Coefficient of variation for $\alpha_{\mathrm{P}}$ versus avalanche angle $\alpha_{\mathrm{P}}$

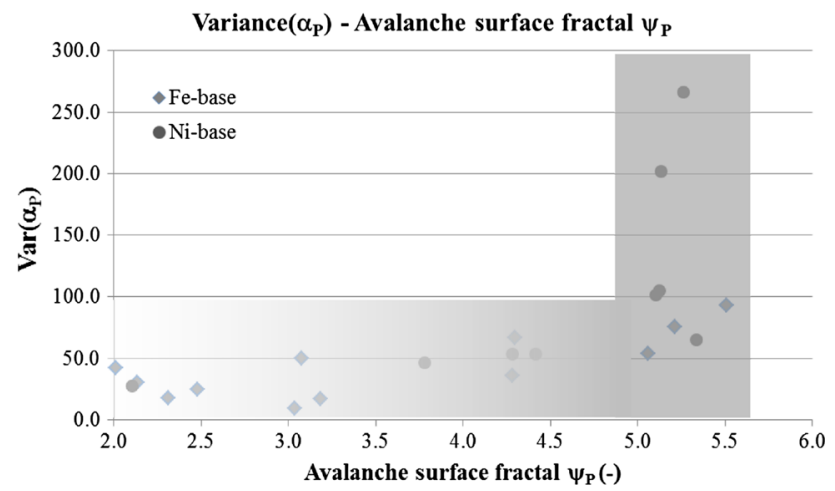

Fig. 11 Variance of the avalanche angle $\operatorname{Var}\left(\alpha_{\mathrm{P}}\right)$ versus avalanche surface fractal $\psi_{\mathrm{P}}$. The darker grey the band the less suitable is a powder for the use in AM

fractal $\psi_{\mathrm{P}}$. The variance is an interesting parameter as it combines $E(f(x))$ with the coefficient of variation $\operatorname{Var} K(f(x))$ as in (5). As good powders will have a low $\alpha_{\mathrm{P}}$ and a low $\operatorname{Var} K\left(\alpha_{\mathrm{P}}\right)$, a low variance will therefore indicate good powders, whereas higher values will be associated with worse powders.

Figure 11 shows that $\operatorname{Var}\left(\alpha_{\mathrm{P}}\right)$ remains comparably low over a wide range of $\psi_{\mathrm{P}}$-values, until about $\psi_{\mathrm{P}}=5.0$, where $\operatorname{Var}\left(\alpha_{\mathrm{P}}\right)$ becomes higher and irregular. The value of $\psi_{\mathrm{P}}=5.0$ indicates therefore a certain limit for good flowable powders. Associated with a $\psi_{\mathrm{P}}$-value of 5.0 is an avalanche angle $\alpha_{\mathrm{P}}$ between $\approx 49^{\circ}(\mathrm{Fe}$ base $)$ and $\approx 54^{\circ}(\mathrm{Ni}$ base), see Fig. 9. The large scatter in Fig. 9, however, does make it difficult to exactly define appropriate limit values for $\psi_{\mathrm{P}}$ and especially $\alpha_{\mathrm{P}}$. In order to quantitatively describe the flowability $\varphi$ of a powder, $\varphi_{\text {opt }}$ is modelled using the most significant flowability parameters. A screening has shown that besides the distribution of flowability parameters $\left(\sigma_{\alpha \mathrm{P}}, \mu_{\psi}, \sigma_{\psi}\right)$ also the particle shape described by ellipticity $E$ does influence the flowability. $\varphi_{\text {opt }}$ is described by:

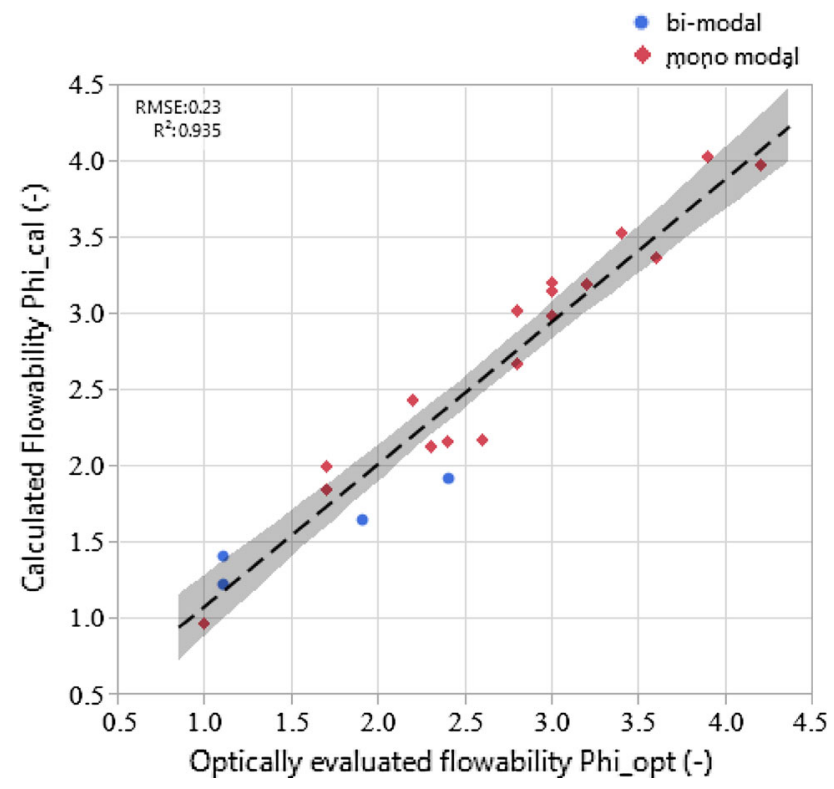

Fig. 12 Predicted flowability $\varphi_{\text {cal }}$ versus observed flowability $\varphi_{\text {opt }}$

$$
\begin{aligned}
\varphi= & 0.843 \times \mu_{\mathrm{SF}}-5.639 \times \sigma_{\mathrm{SF}}+4.144 \times E \\
& -2.669\left(\mu_{\mathrm{SF}}-1.312\right)^{2} \ldots \ldots+9.966\left(\mu_{\mathrm{SF}}-1.312\right) \\
& \times\left(\sigma_{\mathrm{AV}}-0.156\right)-2.758
\end{aligned}
$$

Figure 12 shows that the predicted flowability $\varphi_{\text {cal }}$ is well correlated with the observed flowability $\varphi_{\text {opt }}\left(r^{2}=0.94\right.$, $p<0.0001$, RSME $=0.23$ ). The standard error RSME is in the range of the standard error of the optical evaluations (0.36). It is interesting that Eq. (6) is able to accurately predict the flowability not only for mono-modal, but also for bi-modal powders, as no significant influence of the mode of the PSD can be observed. However, more bimodal powders would be needed to analyse a potential influence in more detail. Additionally, Eq. (6) can predict the flowability for both analysed base materials $\mathrm{Fe}$ and $\mathrm{Ni}$ in a comparable quality as a separate modelling of $\mathrm{Fe}$ and $\mathrm{Ni}$ powders would not lead to much better $r^{2}$ values $\left(r^{2} \approx 0.96\right.$ for both base materials).

\section{Conclusions}

The results indicate that it is possible to distinguish quantitatively between good flowable powders and powders which show insufficient flowability for AM using the Revolution Powder Analyzer. The results correlate with an optical evaluation of powder flowability, which typically is performed today in industry to assess the "suitability" of a powder for AM. A suitable powder therefore is sufficient good to guarantee a homogeneous powder layer quality. However, the flowability parameter $\varphi$ is defined intuitively 
and the quantification underlaid is arbitrary, based on experiences. But, it is an objectively, quantified measure of flowability, allowing to directly compare different powders before the (machine specific) requirements in SLM. Therefore, knowing machine-specific differences, e.g. with regard to the layer creation device, appropriate powder flowability requirements (limits) can be defined. It is proposed to consider this method for standardisation in AM (ASTM, ISO).

Furthermore, it can be expected that $\varphi$ will correlate with other powder layer-specific properties, e.g. powder layer density, and will thereby influence the processing window and the final part properties. This will give further information about appropriate limit values for $\varphi$.

Main effects affecting powder flowability are the distribution of the avalanche surface fractal $\psi_{\mathrm{P}}$ and the avalanche angle $\alpha_{\mathrm{P}}$, which (often) can be described by a lognormal distribution. Additionally, the particle shape (ellipticity) is also an important parameter affecting flowability. This is reasonable as for more elliptical particles the packing density will be reduced to some degree compared to the situation of spherical particles. A looser arrangement of particles leads to a reduction in inter-particle forces (higher distances of the centre of gravity of the particles) and hence to lower $\psi_{\mathrm{P}}$ values. So the surface fractal is considered as a good indirect phenomenological description of these inter-particle forces and therefore significant for the description of powder flowability behaviour. Consequently, $\psi_{\mathrm{P}}$ and $\alpha_{\mathrm{P}}$ will depend on the specific particle size distribution and will serve as additional parameters for the AM qualification of a powder. Additionally, it is expected that for lightweight materials such as aluminium or titanium, there will be an effect of the specific material density, which will be content of future research.

Based on the analysis performed, a quantitative qualification of powders for AM, the flowability $\varphi$ is proposed. It allows to accurately ranking different powders (7). From experience from the assessment of many different powders and for the machine configuration used (ruler and additional powder reservoir next to the build plate), a suitable limit value for $\varphi$ is 2.5 , making sure that values smaller than 2.5 guarantees optically high-quality powder layers in the SLM process. However, this value can be different of other coating devices and machine setups.

$\varphi \leq 2.5$

The measure of powder flowability is, however, only one step in the assessment of the processability of a powder. As shown in Figs. 1 and 2, further requirements have to be defined and quantified to account for other layer properties, including density and the corresponding physical and optical layer properties.

\section{Outlook}

Future work will focus on the correlation between flowability $\varphi$, powder density, the particle size distribution and particle shape. Further work also focuses on the discussion of the influence of different powder parameters on the quality of the SLM parts. Task is to derive a comprehensive set of measurable relevant powder parameters.

Acknowledgments The authors thank Mr. F. Amado for his critically reading this paper and for his helpful advices.

\section{References}

1. Spierings AB, Herres N, Levy G (2011) Influence of the particle size distribution on surface quality and mechanical properties in additive manufactured stainless steel parts. Rapid Prototyp J 17(3):195-202. doi:10.1108/13552541111124770

2. Yasa E, Deckers J, Kruth JP (2011) The investigation of the influence of laser re-melting on density, surface quality and microstructure of selective laser melting parts. Rapid Prototyp J 17(5):312-327

3. Mumtaz KA, Erasenthiran P, Hopkinson N (2008) High density selective laser melting of Waspaloy ${ }^{\circledR}$. J Mater Process Technol 195(1-3):77-87

4. Vandenbroucke B, Kruth JP (2007) Selective laser melting of biocompatible metals for rapid manufacturing of medical parts. Rapid Prototyp J 13(14):196-203. doi:10.1108/ 13552540710776142

5. Brandl E, Heckenberger U, Holzinger V, Buchbinder D (2012) Additive manufactured AlSi10 Mg samples using selective laser melting (SLM): microstructure, high cycle fatigue, and fracture behavior. Mater Des 34:159-169. doi:10.1016/j.matdes.2011.07. 067

6. Buchbinder D, Schleifenbaum H, Heidrich S, Meiners W, Bültmann J (2011) High power selective laser melting (HP SLM) of aluminum parts. Phys Procedia 12:271-278. doi:10.1016/j.phpro. 2011.03.035

7. Boley CD, Khairallah SA, Rubenchik AM (2015) Calculation of laser absorption by metal powders in additive manufacturing. Appl Optics (in press): 11

8. Gürtler FJ, Karg M, Dobler M, Kohl S, Tzivilsky I, Schmidt M (2014) Influence of powder distribution on process stability in laser beam melting: analysis of melt pool dynamics by numerical simulations. In: Bourell D (ed) Solid freeform fabrication symposium. SFF, Austin, pp 1099-1117

9. Boegelein T (2015) Selective laser melting of a ferritic oxide dispersion strengthened steel. Doctoral thesis, University of Liverpool, GB, Liverpool

10. Karapatis NP (2002) A sub-process approach of selective laser sintering. Dissertation, Ecole Polytechnique fédérale de Lausanne EPFL, Lausanne

11. Krantz M, Zhang H, Zhu J (2009) Characterization of powder flow: static and dynamic testing. Powder Technol 194:239-245. doi: 10.1016

12. Castellanos A (2005) The relationship between attractive interparticle forces and bulk behaviour in dry and uncharged fine powders. Adv Phys 54(4):263-276

13. Yu AB, Hall JS (1994) Packing of fine powders subjected to tapping. Powder Technol 78(3):247-256. doi:10.1016/00325910(93)02790-H 
14. Schulze D (1996) Measuring powder flowability: A comparison of test methods. Part I. powder and bulk engineering. CSC Publishing Inc., St Paul

15. Schulze D (1996) Measuring powder flowability: a comparison of test methods. Part II. powder and bulk engineering. CSC Publishing Inc., St Paul

16. ASTM_International (2008) ASTM D6773-08, Standard shear test method for bulk solids using the schulze ring shear tester. ASTM_International, West Conshohocken, PA

17. Schulze D (2011) Flow properties of powders and bulk solids. Ostfalia University of Applied Sciences, Wolfenbüttel

18. Hausner HH (1981) Powder characteristics and their effect on powder processing. Powder Technol 30(1):3-8. doi:10.1016/ 0032-5910(81)85021-8

19. ASTM_International (2009) ASTM D7481-09, standard test methods for determining loose and tapped bulk densities of powders using a graduated cylinder. ASTM_International, West Conshohocken, PA

20. Zocca A, Gomes CM, Mühler T, Günster J (2014) Powder-bed stabilization for powder-based additive manufacturing. Adv Mech Eng 1:6. doi:10.1155/2014/491581

21. Soh JLP, Liew CV, Heng PWS (2006) New indices to characterize powder flow based on their avalanching behavior. Pharm Dev Technol 11(1):93-102. doi:10.1080/10837450500464123

22. Rastogi S, Dhodapkar SV, Cabrejos F, Baker J, Weintraub M, Klinzing GE, Yang WC (1993) Survey of characterization techniques of dry ultrafine coals and their relationships to transport, handling and storage. Powder Technol 74(1):47-59. doi:10.1016/ 0032-5910(93)80007-W

23. Miyajima T, Yamamoto K-I, Sugimoto M (2001) Effect of particle shape on packing properties during tapping. Adv Powder Technol 12(1):117-134. doi:10.1163/156855201745001
24. Zou RP, Yu AB (1996) Evaluation of the packing characteristics of mono-sized non-spherical particles. Powder Technol 88(1):71-79. doi:10.1016/0032-5910(96)03106-3

25. Abdullah EC, Geldart D (1999) The use of bulk density measurements as flowability indicators. Powder Technol 102(2):151-165

26. International Standardisation Organisation (2014) ISO 4490:2014-Metallic powders-Determination of flow rate by means of a calibrated funnel (Hall flowmeter). ISO

27. ASTM_International (2013) ASTM B213-13: standard test methods for flow rate of metal powders using the hall flowmeter funnel. ASTM_International, West Conshohocken, PA

28. ASTM_International (2014) ASTM F3049-14: Standard guide for characterizing properties of metal powders used for additive manufacturing processes. ASTM_International, West Conshohocken, PA

29. Gu H, Gong H, Dilip JJS, Pal D, Hicks A, Doak H (2014) Stucker $\mathrm{BE}$ effects of powder variation on the microstructure and tensile strength of Ti6Al4 V parts fabricated by selective laser melting. In: Bourell D (ed) Solid freeform fabrication symposium. SFF, Austin, pp 470-483

30. Amado F, Schmid M, Levy G, Wegener K (2011) Advances in SLS powder characterization. In: paper presented at the proceedings of the annual international solid freeform fabrication symposium, Austin, Texas, August 3-5

31. Dvorak M, Schmid HG, Fischer F, Barchfeld F (2002) Fast quality control of spray powders/Schnelle Pulverkorngrössenbestimmung zur Qualitätskontrolle. In: International Thermal Spary Conference ITSC, Essen

32. Allen M, Brown GJ, Miles NJ (1995) Measurement of boundary fractal dimensions: review of current techniques. Powder Technol 84(1):1-14. doi:10.1016/0032-5910(94)02967-S 